\title{
Non-Markovian nonstationary completely positive open-quantum-system dynamics
}

\author{
Adrián A. Budini ${ }^{1,2}$ and Paolo Grigolini ${ }^{2,3,4}$ \\ ${ }^{1}$ Consejo Nacional de Investigationes Científicas y Técnicas, Centro Atómico Bariloche, Avenida E. Bustillo Km 9.5, \\ 8400 Bariloche, Argentina \\ ${ }^{2}$ Center for Nonlinear Science, University of North Texas, P.O. Box 311427, Denton, Texas 76203-1427, USA \\ ${ }^{3}$ Istituto dei Processi Chimico Fisici del CNR, Area della Ricerca di Pisa, Via G. Moruzzi, 56124 Pisa, Italy \\ ${ }^{4}$ Dipartimento di Fisica “E. Fermi,” Universitá di Pisa, Largo Pontecorvo 3, 56127 Pisa, Italy
}

(Received 21 April 2009; published 4 August 2009)

\begin{abstract}
By modeling the interaction of a system with an environment through a renewal approach, we demonstrate that completely positive non-Markovian dynamics may develop some unexplored nonstandard statistical properties. The renewal approach is defined by a set of disruptive events, consisting in the action of a completely positive superoperator over the system density matrix. The random time intervals between events are described by an arbitrary waiting-time distribution. We show that, in contrast to the Markovian case, if one performs a system preparation (measurement) at an arbitrary time, the subsequent evolution of the density-matrix evolution is modified. The nonstationary character refers to the absence of an asymptotic master equation even when the preparation is performed at arbitrary long times. In spite this property, we demonstrate that operator expectation values and operators correlations have the same dynamical structure, establishing the validity of a nonstationary quantum regression hypothesis. The nonstationary property of the dynamics is also analyzed through the response of the system to an external weak perturbation.
\end{abstract}

DOI: 10.1103/PhysRevA.80.022103

PACS number(s): 03.65.Yz, 42.50.Lc, 03.65.Ta, 05.40.-a

\section{INTRODUCTION}

The theory of Markovian open-quantum systems [1] is well established from both mathematical and physical points of view. The theory of quantum dynamical semigroups, casting the structure of completely positive $(\mathrm{CP})$ trace-preserving maps, establishes that the Kossakowski-Lindblad equations are the most general admissible forms of evolution of the system density matrix. The application of these equations ranges from quantum optics [2] to quantum information theory [3].

As far as the quantum non-Markovian case is concerned, there exist different physical situations and as a consequence a large variety of formalisms, from which a solid proposal for an approach to non-Markovian quantum dynamics [1,4] may emerge. A promising direction is afforded by the non-Markovian generalization of the Kossakowski-Lindblad equations. In a recent contribution [5], Barnett and Stenholm showed that the adoption of a time convolution between a memory kernel and a Kossakowski-Lindblad operator, although appealing, may lead to unphysical results. However, their attempt attracted the attention of many researchers to the search of the proper memory kernel for the time convoluted Kossakowski-Lindblad equations [6-17]. The main focus of most of these papers has been devoted to the search of memory kernels that guarantees the $\mathrm{CP}$ condition of the solution map [6-14]. On the other hand, different microscopic interactions that lead to the convolution structure were established [15-17] and applied in the characterization of spin environments [18], quantum Boltzmann equations with internal degrees of freedom [19], mesoscopic systems [20], as well as to fluorescent systems coupled to complex selffluctuating environments [21].

It is worth pointing out that the adoption of a renewal approach based on the extension to quantum mechanics of the celebrated continuous-time random walk [22] leads naturally to the time convoluted structure that has been originally hypothesized by the authors of Ref. [5], with no risk of violating the CP condition [7]. In fact, the result is obtained through an average over infinitely many trajectories, each of which consists in a series of sporadic and consecutive transformations (system-environment collisions) of the system density matrix. The $\mathrm{CP}$ condition is trivially satisfied when each collision is written in terms of a CP transformation.

The continuous-time random walk formalism has become a fundamental tool when describing classical (nonMarkovian) complex systems. In particular, the existence of processes without a characteristic time scale (i.e., characterized by power-law behaviors) has lead to an intensive review of the formalism and of its possible extensions. One of the recent motivations for studying that regime comes from the emergence of nonstationary phenomena in the fluorescence intensity produced by (blinking) nanocrystal quantum dots under laser radiation [23-26]. These experiments have led many researchers to revisit some basic tenets and tools of equilibrium and nonequilibrium statistical mechanics, which are closely related to one another: the Onsager principle [27-29], the nonstationary master equations [29], the linearresponse theory [30-38], the Wiener-Khinchin theorem [39], and the ergodic hypothesis [40]. The extension of these principles and theoretical tools for dealing with nonstationary phenomena would be an outstanding breakthrough in statistical mechanics. Besides the theoretical interest, there is an increasing number of experimental situations [41] that can draw benefits from that theoretical progress. While there exist different issues that remain open, we are naturally challenged to find a proper generalization to open-quantumsystem dynamics.

The main goal of this paper is to show that nonMarkovian CP master equations may fit nonstandard nonstationary statistical phenomena and then to analyze the validity 
or the extension of two cornerstones of the theory of openquantum systems, i.e., the quantum regression hypothesis $[2,42]$ and the linear-response theory $[30,31]$. The underlying dynamics of the system are defined by the renewal approach introduced in Ref. [7].

To address the definition (in the context of an openquantum-system theory) of a nonstationary decay (or a nonstationary quantum master equation), we introduce a system preparation at an arbitrary time posterior to the initial coupling between the system and the bath. With preparation, we mean an instantaneous CP operation (like a measurement or any sudden $\mathrm{CP}$ transformation) that leaves the system in an arbitrary state. Then, two times are introduced. One of them $(t)$ [43] measures the time at which the preparation occurs and the second one $(\tau)$ measures the time since the preparation. We shall use the term stationary decay (stationary master equation) to denote a relaxation after the preparation done at time $t$ that is independent of $t$, i.e., it only depends on $\tau$. On the same token, we shall use the term nonstationary decay (nonstationary master equation) to denote relaxation processes whose form depends on $t$, i.e., its functional dependence on $\tau$ is parametrized by $t$. In the Markovian case, the preparation always leads to the same stationary master equation. In the non-Markovian case, we show that, even when the preparation is performed at arbitrary long times, the ensuing relaxation may or may not reach a stationary regime.

We also show that, even in the presence of nonstationary effects, operator expectation values and correlations have the same dynamical structure, providing a generalization of the standard quantum regression theorem $[1,2]$ to a class of nonstationary quantum dynamics. The response of the system to an external weak perturbation, while it can be defined in terms of operator correlations [30,31], generates strong deviations with respect to the Markovian case.

The outline of this paper is as follows. In Sec. II, we review the renewal approach and show how the nonstationary effects arise. In Sec. III we obtain the evolution of both operator expectation values and correlations, which allows us to establish a quantum regression theorem. In Sec. IV, the response to external perturbations is studied. In Sec. V we provide the conclusions.

\section{NONSTATIONARY DENSITY-MATRIX EVOLUTION}

In the quantum application of the renewal approach [7], the density matrix $\rho_{S}(\tau)$ of an open-quantum system $S$ is determined by means of an average over an ensemble of infinitely many stochastic realizations, $\rho_{S}(\tau)=\left\langle\rho_{s t}(\tau)\right\rangle$, where $\langle\cdots\rangle$ denotes the average over realizations and $\rho_{s t}(\tau)$ is the stochastic state associated with each trajectory. They consist of a sequence of disruptive (collisional) events occurring at random times. The times elapsed between two consecutive events are randomly drawn from a waiting-time distribution density $w(t)$, satisfying $w(t) \geq 0$ and $\int_{0}^{\infty} w(t) d t=1$. Each event is associated with an arbitrary $\mathrm{CP}$ transformation $\mathcal{E}$ of the system state. It is defined by the Krauss form [3]

$$
\mathcal{E}[\rho]=\sum_{i} C_{i} \rho C_{i}^{\dagger}
$$

where $\rho$ is the system state prior to a given event. The operators $C_{i}$ satisfy the condition $\Sigma_{i} C_{i}^{\dagger} C_{i}=\mathrm{I}$. Furthermore, here we assume that between consecutive events, the evolution of the system is defined by the propagator $\exp \left[t \mathcal{L}_{S}\right]$. The superoperator $\mathcal{L}_{S}$ is the Liouville superoperator and corresponds to a unitary transformation. Nevertheless, we remark that most of the results hereby developed also apply when $\mathcal{L}_{S}$ is a standard Lindblad superoperator, i.e., when the evolution between events corresponds to a Markovian (CP) dissipative evolution.

By construction, each realization, and as a consequence the average over the realizations, guarantees the $\mathrm{CP}$ condition of the solution map. The system's dynamics begin at time $t=0$ (system-environment coupling). As stressed in the Introduction, we let the system evolve up to the time $t>0$ that we set to be the new origin of time. The earlier work of Ref. [7] is confined to the condition $t=0$ and the main aim of this paper is to solve the nonstationary issues raised by the condition $t>0$. The main idea of the method that we use is as follows. First of all, we study the time evolution of $\rho_{S}$ from $\rho_{S}(0)$ to $\rho_{S}(t+\tau)$, and we interpret the exact expression of $\rho_{S}(t+\tau)$ as the density matrix $\rho_{S}(\tau)$ that will be expressed in terms of the initial condition $\rho(t)$. It is straightforward to get the exact expression of $\rho_{S}(\tau)$, which reads

$$
\rho_{S}(\tau)=\sum_{n=0}^{\infty} \int_{0}^{\tau+t} d t^{\prime} \mathcal{P}_{0}\left(\tau+t-t^{\prime}\right) \mathcal{W}^{(n)}\left(t^{\prime}\right) \rho_{S}(0)
$$

The superoperator $\mathcal{W}^{(n)}(\tau)$ is defined in the Laplace domain $(\tau \rightarrow u, t \rightarrow z)$ as

$$
\mathcal{W}^{(n)}(u) \equiv\left[\mathcal{E} w\left(u-\mathcal{L}_{S}\right)\right]^{n},
$$

while the superoperator $\mathcal{P}_{0}(\tau)$ reads

$$
\mathcal{P}_{0}(u) \equiv P_{0}\left(u-\mathcal{L}_{S}\right),
$$

where $P_{0}(u)$ is the survival probability associated with $w(u)$, i.e.,

$$
P_{0}(u) \equiv \frac{1-w(u)}{u}
$$

The expression given by Eq. (2) is a sum over all possible realizations, each of them corresponding to a stochastic process with $n$ collisions. We have now to express it in terms of the initial condition

$$
\rho_{S}(t)=\sum_{n=0}^{\infty} \int_{0}^{t} d t^{\prime} \mathcal{P}_{0}\left(t-t^{\prime}\right) \mathcal{W}^{(n)}\left(t^{\prime}\right) \rho_{S}(0) .
$$

By using recursively the relation

$$
\begin{aligned}
\mathcal{W}^{(n)}(\tau+t)= & \int_{0}^{\tau} d \tau^{\prime} \mathcal{W}\left(\tau-\tau^{\prime}\right) \mathcal{W}^{(n-1)}\left(\tau^{\prime}+t\right) \\
& +\int_{0}^{t} d t^{\prime} \mathcal{W}\left(\tau+t-t^{\prime}\right) \mathcal{W}^{(n-1)}\left(t^{\prime}\right),
\end{aligned}
$$

we rewrite Eq. (2) as 


$$
\begin{aligned}
\rho_{S}(\tau)= & \Xi_{0}(\tau, t)+\sum_{n=1}^{\infty} \int_{0}^{\tau} d \tau^{\prime} \int_{0}^{\tau^{\prime}} d \tau^{\prime \prime} \mathcal{P}_{0}\left(\tau-\tau^{\prime}\right) \\
& \times \mathcal{W}^{(n-1)}\left(\tau^{\prime}-\tau^{\prime \prime}\right) \Xi_{w}\left(\tau^{\prime \prime}, t\right),
\end{aligned}
$$

where we have defined

$$
\begin{aligned}
& \Xi_{0}(\tau, t)=\sum_{n=0}^{\infty} \int_{0}^{t} d t^{\prime} \mathcal{P}_{0}\left(\tau+t-t^{\prime}\right) \mathcal{W}^{(n)}\left(t^{\prime}\right) \rho_{S}(0), \\
& \Xi_{w}(\tau, t)=\sum_{n=0}^{\infty} \int_{0}^{t} d t^{\prime} \mathcal{W}\left(\tau+t-t^{\prime}\right) \mathcal{W}^{(n)}\left(t^{\prime}\right) \rho_{S}(0) .
\end{aligned}
$$

The Laplace transform of $\rho_{S}(\tau)$ of Eq. (8) then reads

$$
\rho_{S}(u)=\Xi_{0}(u, z)+\sum_{n=1}^{\infty} \mathcal{P}_{0}(u) \mathcal{W}^{(n-1)}(u) \Xi_{w}(u, z) .
$$

By using the relation

$$
\int_{0}^{\infty} d \tau \int_{0}^{\infty} d t e^{-u \tau} e^{-z t} g(\tau+t)=\frac{g(u)-g(z)}{z-u},
$$

which is valid for any arbitrary function $g(t)$, with the notations $g(u) \equiv \int_{0}^{\infty} d \tau e^{-u \tau} g(\tau)$ and $g(z) \equiv \int_{0}^{\infty} d t e^{-z t} g(t)$, the Laplace transforms of $\Xi_{0}(\tau, t)$ and $\Xi_{w}(\tau, t)$ are written as

$$
\begin{aligned}
& \Xi_{0}(u, z)=\frac{\mathcal{P}_{0}(u)-\mathcal{P}_{0}(z)}{z-u} \frac{1}{\mathcal{P}_{0}(z)} \rho_{S}(z), \\
& \Xi_{w}(u, z)=\frac{\mathcal{W}(u)-\mathcal{W}(z)}{z-u} \frac{1}{\mathcal{P}_{0}(z)} \rho_{S}(z) .
\end{aligned}
$$

Here, $\rho_{S}(z)$ is the Laplace transform of $\rho_{S}(t)$ given by Eq. (6). By plugging these expressions into Eq. (11), after some algebra we get

$$
\rho_{S}(u)=\mathcal{G}(u)\left[\rho_{S}(z)+\mathrm{I}_{\rho}(u, z)\right],
$$

where the propagator $\mathcal{G}(u)$ is defined by

$$
\mathcal{G}(u)=\frac{1}{u-\left[\mathcal{L}_{S}+\mathcal{L} K\left(u-\mathcal{L}_{S}\right)\right]},
$$

and the inhomogeneous term is defined by

$$
\mathrm{I}_{\rho}(u, z)=\mathcal{L} \Delta\left(u-\mathcal{L}_{S}, z-\mathcal{L}_{S}\right)\left(z-\mathcal{L}_{S}\right) \rho_{S}(z) .
$$

In the time domain, Eq. (15) becomes

$$
\begin{aligned}
\frac{d \rho_{S}(\tau)}{d \tau}= & \mathcal{L}_{S} \rho_{S}(\tau)+\int_{0}^{\tau} d \tau^{\prime} K\left(\tau-\tau^{\prime}\right) \mathcal{L} e^{\left(\tau-\tau^{\prime}\right) \mathcal{L}_{S}} \rho_{S}\left(\tau^{\prime}\right) \\
& +\mathrm{I}_{\rho}(\tau, t)
\end{aligned}
$$

This equation is one of the central results of this section. It defines the evolution of the average density matrix of the system in the interval $(t, t+\tau)$, with the initial condition (6). The superoperator $\mathcal{L}$ is defined by

$$
\mathcal{L}=\mathcal{E}-1,
$$

which in turn can be written with the Lindblad structure

$$
\mathcal{L}[\bullet]=\frac{1}{2} \sum_{i}\left\{\left[C_{i}, \bullet C_{i}^{\dagger}\right]+\left[C_{i} \bullet, C_{i}^{\dagger}\right]\right\} .
$$

The memory kernel function $K(\tau)$ is defined in the Laplace domain by

$$
K(u)=\frac{u w(u)}{1-w(u)} .
$$

The inhomogeneous contribution $\mathrm{I}_{\rho}(u, z)[\mathrm{Eq} .(17)]$ is proportional to the function

$$
\Delta(u, z)=\frac{\tilde{w}(u, z)}{1-w(u)}-\frac{w(u) / z}{1-w(u)},
$$

where the function $\tilde{w}(u, z)$ reads

$$
\widetilde{w}(u, z)=\frac{w(u)-w(z)}{z-u} \frac{1}{1-w(z)} .
$$

By using the relation of Eq. (12), $\widetilde{w}(u, z)$ can be written in the time domain as

$$
\widetilde{w}(\tau, t)=w(\tau+t)+\sum_{n=1}^{\infty} \int_{0}^{t} d t^{\prime} w\left(\tau+t-t^{\prime}\right) w^{(n)}\left(t^{\prime}\right),
$$

where $w^{(n)}(z) \equiv[w(z)]^{n}$. This expression allows us to interpret $\widetilde{w}(\tau, t)$ as a conditional waiting-time distribution density and more precisely as the probability distribution density of meeting the first event at time $\tau$, given that the observation time (of events) begins at time $t$. The second term on the right-hand side of Eq. (24) takes into account all possible events at times earlier than $t$. Consistently, notice that, for $t$ $=0, \widetilde{w}(\tau, 0)=w(\tau)$.

In the time domain, the function $\Delta(u, z)$ reads

$$
\Delta(\tau, t)=f(\tau, t)-f(\tau, 0),
$$

where we have introduced the (sprinkling) distribution

$$
f(u, z)=\frac{\widetilde{w}(u, z)}{1-w(u)} .
$$

By writing this expression in the time domain,

$$
f(\tau, t)=\tilde{w}(\tau, t)+\sum_{n=1}^{\infty} \int_{0}^{\tau} d \tau^{\prime} w^{(n)}\left(\tau-\tau^{\prime}\right) \widetilde{w}\left(\tau^{\prime}, t\right),
$$

it follows that $f(\tau, t) d \tau$ is the probability of an event occurrence in the time interval $(\tau, \tau+d \tau)$, given that the observation time begins at time $t$, regardless of whether or not any event occurred at earlier times. It satisfies the relation $f(\tau, 0)=\widetilde{w}(0, \tau)$. On the other hand, notice that the function $\Upsilon(u, z) \equiv z \Delta(u, z)$ [appearing in Eq. (17)] can be written in the time domain as $Y(\tau, t)=(d / d t) f(\tau, t)$.

Both the kernel $K(\tau)$ and the inhomogeneous contribution $\mathrm{I}_{\rho}(\tau, t)$ are clear signatures of the non-Markovian property of the evolution equation (18). Using Eq. (15), it is easy to realize that the evolution of $\rho_{S}(\tau)$ can always be rewritten as a homogeneous evolution [see, for example, Eq. (31)]. Nevertheless, the inhomogeneous structure allows us to understand what is the effect of shifting the initial time condition from $\rho_{S}(0)$ to $\rho_{S}(t)$. In fact, Eq. (17) tells us that in the 
interval $(t, t+\tau)$ the departure of the system time evolution from its time evolution in $(0, t)$ is measured by Eq. (25). Consistently, for $t=0$, the inhomogeneous contribution vanishes, i.e., $\mathrm{I}_{\rho}(\tau, 0)=0$.

No departure of the system evolution in $(t, t+\tau)$ from the time evolution in $(0, t)$ must occur in the Markovian case. This case is recovered by assuming an exponential waitingtime distribution (Poisson case), $w(\tau)=\gamma \exp [-\gamma \tau]$. From Eqs. (23) and (26), it follows that $\widetilde{w}(\tau, t)=w(\tau)$ and $f(\tau, t)$ $=\gamma$, thereby implying the vanishing of the inhomogeneous term, and the relation $K(\tau)=\gamma \delta(\tau)$, which turns Eq. (18) into a standard Lindblad equation

$$
\frac{d \rho_{S}(\tau)}{d \tau}=\left[\mathcal{L}_{S}+\gamma \mathcal{L}\right] \rho_{S}(\tau) .
$$

On the other hand, we remark that, in Eq. (18), and in Eq. (17) as well, the superoperator $\mathcal{L}$ may taken as an arbitrary Lindblad form. In fact, when $\mathcal{L} \neq \mathcal{E}-\mathrm{I}$ [see Eq. (19)] the superoperator $\mathcal{E}\left[\right.$ Eq. (1)] can be defined as $\mathcal{E}[\rho]=\left\{\mathrm{I}+\left[e^{\kappa \mathcal{L}}\right.\right.$ $-\mathrm{I}]\} \rho$, where $\kappa$ must be interpreted as a control parameter. Then, we recover Eq. (18) with an arbitrary $\mathcal{L}$ in the limiting condition, in which simultaneously $\kappa \rightarrow 0$ and the number of events per unit of time goes to infinity, with the last limit being controlled by the distribution $f(\tau, 0)$ of Eq. (27).

\section{A. Initial preparation at time $t$}

The initial condition associated with Eq. (18) is given by $\rho_{S}(t)$, Eq. (6), which in turn carries information about the system dynamics in the interval $(0, t)$. Therefore, Eq. (18) does not give more information than a master equation describing the evolution in the interval $(0, t+\tau)$. Nevertheless, the master equation (18) may acquire a different status if the initial condition at time $t$ can be chosen as any nonequilibrium form of the density matrix $\rho_{S}$. This is done by introducing the main ingredient of our formalism, i.e., by adopting

ПI

the concept of preparation, namely, a change $\rho_{S}(t) \rightarrow \rho_{\Pi}$, compatible with a $\mathrm{CP}$ transformation $\Pi$. The role of the preparation is to erase the dependence of the evolution on the previous history of the system without erasing the memory of the universe, i.e., the system-environment arrangement.

In the Laplace domain the preparation is defined by

$$
\left(z-\mathcal{L}_{S}\right) \rho_{S}(z) \rightarrow \rho_{\Pi},
$$

which in the time domain yields $\rho_{S}(t) \rightarrow \rho_{\Pi} \exp \left[\mathcal{L}_{S} t\right]$. The extra unitary contribution is introduced to take into account that the Hamiltonian evolution defined by $\mathcal{L}_{S}$ begins at time $t=0$. Similarly, one can interpret Eq. (29) as a preparation in an interaction representation with respect to $\mathcal{L}_{S}$.

Under the preparation condition of Eq. (29), the time evolution structure of Eq. (18) is still valid provided that the initial condition is fixed to be $\rho_{\Pi} \exp \left[\mathcal{L}_{S} t\right]$, with the inhomogeneous term now reading

$$
\mathrm{I}_{\rho}(\tau, t)=\mathcal{L} \Delta(\tau, t) \exp \left[(\tau+t) \mathcal{L}_{S}\right] \rho_{\Pi} .
$$

We remark that the time evolution of Eq. (18) with the contribution of Eq. (30) remains a CP structure. In fact, also its solution admits an interpretation in terms of trajectories that preserve the $\mathrm{CP}$ condition.

The time evolution of the density matrix generated by Eq. (18), with the inhomogeneous contribution of Eq. (30), in principle depends on the preparation time $t$. What is the form of the dependence of the density time evolution in $(t, t+\tau)$ on $t$ ? Of particular interest is to assess under what conditions this dependence is lost, so as to generate in the long-time limit the stationary behavior defined in Sec. I. In the case where the solution becomes asymptotically stationary, it is of interest to assess if this stationary time evolution $(t \rightarrow \infty)$ is characterized by non-Markovian effects stronger or weaker than the time evolution with the preparation stage coinciding with the initialization stage, i.e., with $t=0$. These important questions will be answered with the help of the simple examples discussed in Sec. II B.

As the last but not the least remark of this section, let us notice that the time evolution of Eq. (18), with the preparation condition of Eq. (29), can be easily written in an equivalent form, as a homogeneous time evolution, as follows:

$$
\begin{aligned}
\frac{d \rho_{S}(\tau)}{d \tau}= & \mathcal{L}_{S} \rho_{S}(\tau)+\int_{0}^{\tau} d \tau^{\prime} \int_{0}^{\tau^{\prime}} d \tau^{\prime \prime} \\
& \times \mathcal{M}_{t}\left(\tau-\tau^{\prime}\right) K_{t}\left(\tau^{\prime}-\tau^{\prime \prime}\right) \mathcal{L} e^{\left(\tau^{\prime}-\tau^{\prime \prime}\right) \mathcal{L}_{S}} \rho_{S}\left(\tau^{\prime \prime}\right),
\end{aligned}
$$

where $\quad \mathcal{M}_{z}(u)=\left[1+\mathcal{L} \Delta\left(u-\mathcal{L}_{S}, z-\mathcal{L}_{S}\right)\right]^{-1} \quad$ and $\quad K_{t}(u)$ $=u \widetilde{w}(u, t) /[1-w(u)]$. While this expression avoids the complication arising from the presence of an inhomogeneous term, the kernel structure is more complicated, involving all powers of the Lindblad superoperator $\mathcal{L}$. Equation (31) recovers and generalizes the classical master equation obtained in Ref. [29].

\section{B. Examples}

To make the spirit of the renewal approach of this paper more transparent, here, we illustrate it in action on two exemplary cases of the same simple model. While the microscopic origin the superoperator $\mathcal{E}$ and the waiting-time distribution $w(t)$ are not completely understood $[7,15]$, from Eq. (18) it becomes clear that the former object defines the underlying Lindblad-like structure (20). Then, it establishes the coupling between the density-matrix elements. On the other hand, $w(t)$ can be settled in a phenomenological way as a function of the characteristic system decay behavior (see next examples). Its leading property is the average waiting time $\int_{0}^{\infty} t w(t) d t$, which may be finite or divergent, with the last case giving rise to strong nonstationary effects.

As a simple model, we consider a degenerate two-level system $\left(\mathcal{L}_{S} \rightarrow 0\right)$ and the superoperator

$$
\mathcal{E}[\bullet]=\sigma_{z} \cdot \sigma_{z}
$$

The time evolution of the expectation values of the Pauli matrixes $\sigma_{i}, S_{i}(\tau) \equiv \operatorname{Tr}_{S}\left[\rho_{S}(\tau) \sigma_{i}\right]$, with $i=x, y, z$, is given by Eqs. (18) and (30) [or equivalently by Eq. (31)], and it reads 


$$
\frac{d S_{X, Y}(\tau)}{d \tau}=-\int_{0}^{\tau} d \tau^{\prime} \tilde{K}_{t}\left(\tau-\tau^{\prime}\right) S_{X, Y}\left(\tau^{\prime}\right),
$$

while $S_{Z}(\tau)=S_{Z}(0)$. With $S_{i}(0)$, we denote the expectation values after the preparation. The kernel is defined by its Laplace transform $\widetilde{K}_{t}(u)=u \widetilde{w}(u, t) /[1-\widetilde{w}(u, t)]$. The solution of Eq. (33) is

$$
S_{X, Y}(\tau)=S_{X, Y}(0) \tilde{P}_{0}(\tau, t),
$$

where $\widetilde{P}_{0}(\tau, t)$ is the survival probability associated with $\widetilde{w}(\tau, t)$, i.e., $\widetilde{P}_{0}(\tau, t)=1-\int_{0}^{\tau} d \tau^{\prime} \widetilde{w}\left(\tau^{\prime}, t\right)$. It can be rewritten as

$$
\tilde{P}_{0}(\tau, t)=P_{0}(\tau+t)+\int_{0}^{t} d t^{\prime} P_{0}\left(\tau+t-t^{\prime}\right) f\left(t^{\prime}, 0\right) .
$$

Here, $P_{0}(\tau)$ is defined by its Laplace transform (5), while $f(t, 0)$ follows from Eq. (27), i.e., $f(z, 0)=w(z) /[1-w(z)]$.

The decay of the expectation values of Eq. (34) depends on both $\tau$ and $t$. Its explicit analytical form depends on the choice done for the waiting-time distribution $w(t)$. As first case, we select the biexponential case

$$
w(t)=P_{a} \gamma_{a} e^{-\gamma_{a} t}+P_{b} \gamma_{b} e^{-\gamma_{b} t},
$$

with $P_{a}+P_{b}=1$. Distribution (27) $(t=0)$ reads

$$
f(\tau, 0)=\langle\gamma\rangle \theta(\tau)-\left[\langle\gamma\rangle-\langle\tau\rangle^{-1}\right]\left(1-e^{-\eta \tau}\right),
$$

where $\theta(\tau)$ is the step function and we have introduced the parameters $\langle\gamma\rangle \equiv P_{a} \gamma_{a}+P_{b} \gamma_{b}, \quad\langle\tau\rangle \equiv P_{a} \gamma_{a}^{-1}+P_{b} \gamma_{b}^{-1}$ $=\int_{0}^{\infty} \tau w(\tau) d \tau<\infty$, and $\eta \equiv P_{a} \gamma_{b}+P_{b} \gamma_{a}$. Notice that after a transient of order $1 / \eta$, the sprinkling distribution, as in the Markovian case, is constant, i.e., $f(\tau, 0) \simeq 1 /\langle\tau\rangle>0$.

The coherence decay, independent of the time $t$, can be written as

$$
\widetilde{P}_{0}(\tau, t)=P_{a}(t) e^{-\gamma_{a} \tau}+P_{b}(t) e^{-\gamma_{b} \tau} .
$$

All the dependence on the preparation time is carried out by the weights $P_{a}(t)$ and $P_{b}(t)$. Their explicit form follows straightforwardly from Eq. (35) as a superposition of exponential functions. They satisfy the boundary conditions $P_{a}(0)=P_{a}$ and $P_{b}(0)=P_{b}$. In the limit $t \rightarrow \infty$, the asymptotic stationary decay reads

$$
\widetilde{P}_{0}(\tau, \infty)=\frac{P_{a}}{\langle\tau\rangle \gamma_{a}} e^{-\gamma_{a} \tau}+\frac{P_{b}}{\langle\tau\rangle \gamma_{b}} e^{-\gamma_{b} \tau} .
$$

In Fig. 1, we show the decay defined by $\widetilde{P}_{0}(\tau, t)$ for different preparation times $t$ and for two different sets of characteristic parameter values. After a transient of order $\eta$, both cases reach a stationary decay regime. By comparing these figures with the other, we realize that the asymptotic decay may yield arbitrary departures from the dynamics generated by setting $t=0$. In fact, in Fig. 1(a), the asymptotic decay is almost exponential while the initial one is biexponential. In Fig. 1(b) the inverse situation is observed. This simple example demonstrates that no general conclusion can be drawn about the properties of the stationary time evolution.

As a second exemplary case, we consider the waitingtime distribution
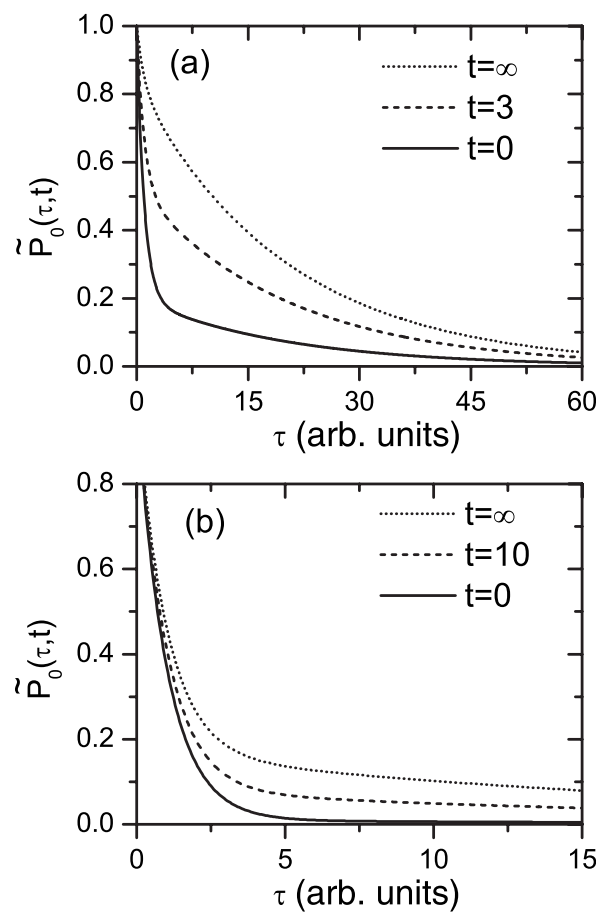

FIG. 1. Coherence decay $\widetilde{P}_{0}(\tau, t)$ [Eq. (34)], for different times $t$, for the waiting-time distribution (36). In (a) the parameters are $P_{a}=0.8$ and $P_{b}=0.2$. In (b) the parameters are $P_{a}=0.99$ and $P_{b}$ $=10^{-3}$. In both cases the rates are $\gamma_{a}=1$ and $\gamma_{b}=0.05$. Both $\tau$ and $t$ are measured in arbitrary units.

$$
w(u)=\frac{A_{\alpha}}{A_{\alpha}+u^{\alpha}} .
$$

where the units of $A_{\alpha}$ are $1 / \mathrm{s}^{\alpha}$, and $0<\alpha \leq 1$. Note that for $\alpha=1$ this expression reduces to the Laplace transform of an exponential function. Kernel (21) reads $K(u)=A_{\alpha} u^{1-\alpha}$. As is well known [44], this kind of kernel is related to a fractional derivative operator. In contrast to Eq. (37), here we get

$$
f(\tau, 0)=\frac{A_{\alpha}}{\Gamma(\alpha)} \frac{1}{\tau^{(1-\alpha)}},
$$

where $\Gamma(x)$ is the gamma function. Then, in this case, $\lim _{\tau \rightarrow \infty} f(\tau, 0)=0$. This property is directly related to the divergence of the average period between events, i.e., $\int_{0}^{\infty} \tau w(\tau) d \tau=\infty[44]$.

By using Eq. (35) and the fact that $P_{0}(\tau)$ is, in this case, a Mittag-Leffler function $[7,44]$, we can write $\widetilde{P}_{0}(\tau, t)[45]$ under the form of a series expansion. By using the property that, for $A_{\alpha} \tau^{\alpha} \gg 1, P_{0}(\tau) \approx A_{\alpha} /\left[\tau^{\alpha} \Gamma(1-\alpha)\right]$, when $\tau \gg t$ we get the following asymptotic expression:

$$
\widetilde{P}_{0}(\tau, t) \approx \frac{1}{\Gamma(1-\alpha)}\left[\frac{A_{\alpha}^{-1}}{(\tau+t)^{\alpha}}+\frac{1}{\alpha \Gamma(\alpha)} \frac{t^{\alpha}}{(\tau+t)^{\alpha}}\right] .
$$

Therefore, in this case there not exists an asymptotic stationary decay. In fact, this expression shows that at any time the decay dynamics depends on the preparation time $t$. In Fig. 2 we plot the function $\widetilde{P}_{0}(\tau, t)$, Eq. (35), for different values of $t$. Equation (42) correctly fits their asymptotic decay behav- 


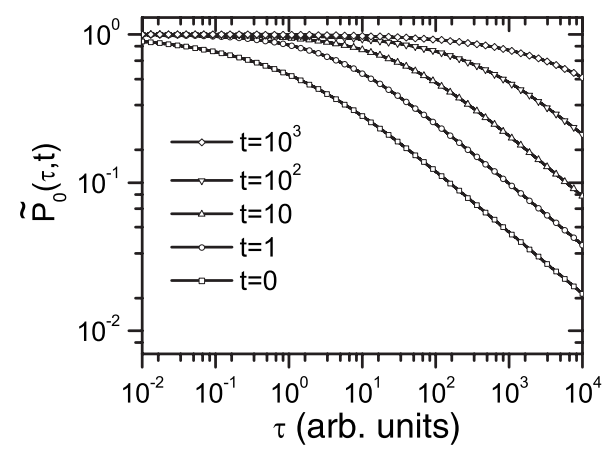

FIG. 2. Coherence decay $\widetilde{P}_{0}(\tau, t)$ [Eq. (34)], for different times $t$, for the fractional waiting-time distribution (40). The parameters are $A_{\alpha}=1$ and $\alpha=1 / 2$. Both $\tau$ and $t$ are measured in arbitrary units.

ior. Consistently, we found that there not exists a stationary decay behavior.

\section{REGRESSION HYPOTHESIS}

The generalization of the classical regression hypothesis [27] to a quantum context is called quantum regression theorem $[2,42]$. It states that operator expectation values and operator correlations have the same dynamical behavior. Here, we explore the possibility of generalizing this theorem to the renewal case.

\section{A. Operator dual evolution}

In order to define operator correlations, we have to move from the Schrödinger to the dual or Heisenberg representation. In the renewal case here under study, this corresponds to converting the stochastic time evolution of the density matrix $\rho_{s t}(t)$ into the stochastic time evolution of operators. All of these rest on the fundamental relation

$$
\overline{A(t)}=\operatorname{Tr}_{S}\left[A(0) \rho_{S}(t)\right]=\operatorname{Tr}_{S}\left[\rho_{S}(0) A(t)\right],
$$

where the mean value $\overline{A(t)}$ of a system operator $A$ can be written in terms of the initial density matrix $\rho_{S}(0)$ and of the evolved operator $A(t)$.

Let us define the dual superoperators $\mathcal{L}_{S}^{\#}$ and $\mathcal{E}^{\#}$ by the relations

$$
\begin{gathered}
\operatorname{Tr}_{S}\left[A e^{t \mathcal{L}_{S}} \rho\right]=\operatorname{Tr}_{S}\left[\rho e^{t \mathcal{L}_{S A}^{\#}}\right], \\
\operatorname{Tr}_{S}[A \mathcal{E} \rho]=\operatorname{Tr}_{S}\left[\rho \mathcal{E}^{\#} A\right] .
\end{gathered}
$$

Equation (2) yields the (averaged over realizations) operator time evolution

$$
A(t+\tau)=\sum_{n=0}^{\infty} \int_{0}^{\tau+t} d t^{\prime} \mathcal{W}^{\sharp(n)}\left(t^{\prime}\right) \mathcal{P}_{0}^{\#}\left(\tau+t-t^{\prime}\right) A(0) .
$$

Here, $\mathcal{W}^{\#(n)}(z)=\left[w\left(z-\mathcal{L}_{S}^{\#}\right) \mathcal{E}^{\#}\right]^{n}$ arises from Eq. (3) and $\mathcal{P}_{0}^{\#}(z)=P_{0}\left(z-\mathcal{L}_{S}^{\#}\right)$ arises from Eq. (5). Note that, as a consequence of the prescription of Eq. (43), the $\mathcal{W}^{\#}$ superoperator applies after the $\mathcal{P}_{0}^{\#}$ one rather than before it as in Eq. (2).

In conclusion the dynamics of the stochastic operator $A_{s t}$ resembles that of the stochastic density matrix $\rho_{s t}$. It consists, too, of time intervals with the time evolution driven by $\exp \left[t \mathcal{L}_{S}^{\#}\right]$, and of others, corresponding to the action of the superoperator $\mathcal{E}^{\#}$, where it is driven by disruptive events. Nevertheless, notice that, when $\left[\mathcal{L}_{S}^{\#}, \mathcal{E}^{\#}\right] \neq 0$, the time ordering of the superoperators is reversed as a consequence of turning the Schrödinger's into the Heisenberg's representation.

\section{B. Operator expectation values and correlations}

For the main purpose of working with simplified expressions, in this section we make all calculations in the interaction representation with respect to $\mathcal{L}_{S}^{\#}$, and we assume that

$$
\left[\mathcal{L}_{S}^{\#}, \mathcal{E}^{\#}\right]=0
$$

which in turn in the Schrödinger representation yields $\left[\mathcal{L}_{S}, \mathcal{E}\right]=0$ or, equivalently, the commutation condition $\left[\mathcal{L}_{S}, \mathcal{L}\right]=0$. As a consequence of this condition, Eq. (45) $[\mathrm{Eq}$. (2)], it follows that the operator [density-matrix] stochastic dynamics, in the interaction representation with respect to $\mathcal{L}_{S}^{\#}$ $\left[\mathcal{L}_{S}\right]$, only consists in the application of the superoperator $\mathcal{E}^{\sharp}$ $[\mathcal{E}]$ at random times. Notice that all the expressions obtained in the previous section, in the interaction representation, remain valid by setting $\mathcal{L}_{S} \rightarrow 0$.

The operator expectation values, in the interval $(t, t+\tau)$, are written as $[\overline{\mathbf{A}(\tau+t)} \rightarrow \overline{\mathbf{A}(\tau)}]$,

$$
\begin{aligned}
\overline{\mathbf{A}(\tau)} & =\operatorname{Tr}_{S}\left[\rho_{S}(0) \mathbf{A}(\tau+t)\right], \\
& =\operatorname{Tr}_{S}\left[\rho_{S}(0) C_{\mathrm{IA}}(\tau, t)\right] .
\end{aligned}
$$

With $\mathbf{A}=\left(A_{1}, A_{2}, \ldots\right)^{T}$, we denote a vector of system operators defining a complete basis in the dual (operators) space. I denotes the system identity operator. The operator correlations are written as

$$
\begin{aligned}
\overline{O(t) \mathbf{A}(t+\tau)} & =\operatorname{Tr}_{S}\left[\rho_{S}(0) O(t) \mathbf{A}(t+\tau)\right], \\
& =\operatorname{Tr}_{S}\left[\rho_{S}(0) C_{O \mathbf{A}}(\tau, t)\right] .
\end{aligned}
$$

Here, $O$ denotes an arbitrary system operator. The auxiliary operator functions $C_{U V}(\tau, t)$, acting on arbitrary system operators $U$ and $V$, are defined by the expression

$$
C_{U V}(\tau, t) \equiv \sum_{m=0}^{\infty} \sum_{n=0}^{\infty} P(\tau, m ; t, n)\left(\mathcal{E}^{\#}\right)^{n}\left[U\left(\mathcal{E}^{\#}\right)^{m}[V]\right] .
$$

$P(\tau, m ; t, n)$ is the probability that $n$ events occur in the interval $(0, t)$ and that $m$ events occur in the interval $(t, t+\tau)$. Under condition (46), in the interaction representation with respect to $\mathcal{L}_{S}^{\#}$, Eq. (49) follows straightforwardly from the stochastic dynamics associated with Eq. (45).

The set of probabilities $P(\tau, m ; t, n)$ can be written as

$$
P(\tau, 0 ; t, n)=\int_{0}^{t} d t^{\prime} P_{0}\left(\tau+t-t^{\prime}\right) w^{(n)}\left(t^{\prime}\right),
$$

when $m=0$, and as 


$$
\begin{aligned}
P(\tau, m ; t, n)= & \int_{0}^{\tau} d \tau^{\prime} P_{0}\left(\tau-\tau^{\prime}\right) \\
& \times \int_{0}^{\tau^{\prime}} d \tau^{\prime \prime} w^{(m-1)}\left(\tau^{\prime}-\tau^{\prime \prime}\right) \\
& \times \int_{0}^{t} d t^{\prime} w\left(\tau^{\prime \prime}+t-t^{\prime}\right) w^{(n)}\left(t^{\prime}\right),
\end{aligned}
$$

for $m \geq 1$. As before, the function $w^{(n)}(\tau)$ is defined by its Laplace transform $w^{(n)}(u)=[w(u)]^{n}$. In the Laplace domain $(\tau \rightarrow u, t \rightarrow z)$, after using Eq. (12), we get

$$
P(u, 0 ; z, n)=\frac{1-z \widetilde{w}(u, z)}{u} P_{0}(z)[w(z)]^{n},
$$

and for $m \geq 1$,

$$
P(u, m ; z, n)=P_{0}(u)[w(u)]^{m-1} z \widetilde{w}(u, z) P_{0}(z)[w(z)]^{n},
$$

where $\widetilde{w}(u, z)$ is defined by Eq. (23).

After some algebra based on Eq. (49) we write

$$
C_{U V}(u, z)=\mathcal{G}^{\#}(z) U \mathcal{G}^{\#}(u)\left[1+z \Delta(u, z) \mathcal{L}^{\#}\right] V .
$$

The function $\Delta(u, z)$ is defined by Eq. (22) and $\mathcal{G}^{\#}(u)$ denotes the propagator

$$
\mathcal{G}^{\#}(u)=\frac{1}{u-K(u) \mathcal{L}^{\#}},
$$

where $\mathcal{L}^{\#} \equiv \mathcal{E}^{\#}-1$ is the dual superoperator associated with $\mathcal{L}$ [Eq. (19)]. The kernel $K(u)$ follows from Eq. (21). Therefore, taking into account that these expressions were derived in an interaction representation with respect to $\mathcal{L}_{S}$, we obtain that $\mathcal{G}^{\#}(u)$ is the dual propagator associated with $\mathcal{G}(u)$ [Eq. (16)].

From Eq. (52), after introducing the density matrix

$$
\rho_{S}(z)=\mathcal{G}(z) \rho_{S}(0),
$$

the mean values (47) and correlations (48) read

$$
\begin{gathered}
\overline{\mathbf{A}(\tau)} \doteq \operatorname{Tr}_{S}\left\{\rho_{S}(z) \mathcal{G}^{\#}(u)\left[1+z \Delta(u, z) \mathcal{L}^{\#}\right] \mathbf{A}\right\}, \\
\overline{O(t) \mathbf{A}(t+\tau)} \doteq \operatorname{Tr}_{S}\left\{\rho_{S}(z) O \mathcal{G}^{\#}(u)\left[1+z \Delta(u, z) \mathcal{L}^{\#}\right] \mathbf{A}\right\} .
\end{gathered}
$$

Here, for the sake of shortening the notation, we use the symbol $\doteq$ to indicate that the left- and the right-hand sides of the equality are written in the time and the Laplace domains, respectively. These equations yield the desired expressions for operator expectation values and correlations. They can be straightforwardly written in terms of density-matrix propagators as

$$
\begin{gathered}
\overline{\mathbf{A}(\tau)} \doteq \operatorname{Tr}_{S}\left\{\mathbf{A} \mathcal{G}(u)[1+\mathcal{L} \Delta(u, z) z] \rho_{S}(z)\right\}, \\
\overline{O(t) \mathbf{A}(t+\tau)} \doteq \operatorname{Tr}_{S}\left\{\mathbf{A} \mathcal{G}(u)[1+\mathcal{L} \Delta(u, z) z] \rho_{S}(z) O\right\} .
\end{gathered}
$$

In the Markov case, i.e., when $K(u)=\gamma$, these results recover the expressions that follows from a microscopic derivation based on a Born-Markovian approximation [2]. Furthermore, by using the same calculations steps, it is possible to demonstrate that

$$
\overline{O(t) \mathbf{A}(t+\tau) \tilde{O}(t)} \doteq \operatorname{Tr}_{S}\left\{\mathbf{A} \mathcal{G}(u)[1+z \Delta(u, z) \mathcal{L}] \tilde{O} \rho_{S}(z) O\right\}
$$

From Eqs. (55) and (56), it is immediate to realize that expectation values and correlations have the same dynamical structure, showing that the classical Onsager regression hypothesis can be extended to this context. We make this fact even clearer by introducing a preparation at time $t$ [Eq. (29)], thereby implying the transformation $z \rho_{S}(z) \rightarrow \rho_{\Pi}$. Then, the preparation can be interpreted as a sudden fluctuation at time $t$. The earlier expressions indicate that the operator correlation dynamics depend on the dynamical decay of this fluctuation.

\section{Evolutions}

We can explicitly show that operator expectation values and correlations have the same dynamical behavior. Here, we obtain the inhomogeneous equations of motion. Nevertheless, as in Eq. (31), they can be rewritten as homogeneous ones. By defining a matrix $\mathbb{M}$ by the relation

$$
\operatorname{Tr}_{S}[\mathbf{A} \mathcal{L} O]=\mathbb{M} \operatorname{Tr}_{S}[\mathbf{A} O],
$$

which acts on the indices of vector $\mathbf{A}$, from Eq. (55) it is possible to get the evolution

$$
\frac{d}{d \tau} \overline{\mathbf{A}(\tau)}=\int_{0}^{\tau} d t^{\prime} K\left(\tau-\tau^{\prime}\right) \mathrm{M} \overline{\mathbf{A}\left(\tau^{\prime}\right)}+\Gamma_{\mathrm{IA}}(\tau, t),
$$

while from Eq. (56), for the correlations it follows that

$$
\begin{aligned}
\frac{d}{d \tau} \overline{O(t) \mathbf{A}(t+\tau)}= & \int_{0}^{\tau} d \tau^{\prime} K\left(\tau-\tau^{\prime}\right) \bar{M} \overline{O(t) \mathbf{A}\left(t+\tau^{\prime}\right)} \\
& +\Gamma_{O \mathbf{A}}(\tau, t)
\end{aligned}
$$

The inhomogeneous terms $\Gamma_{\mathrm{IA}}(\tau, t)$ and $\Gamma_{O \mathbf{A}}(\tau, t)$, taking into account the preparation [Eq. (29)], follow from

$$
\Gamma_{O A}(\tau, t)=\Delta(\tau, t) \mathbb{M} \operatorname{Tr}_{S}\left[O \mathbf{A} \rho_{\Pi}\right] .
$$

These expressions show that even in the presence of strong non-Markovian nonstationary effects, the regression hypothesis is still valid [28,29].

\section{Discussion}

The previous analysis demonstrates that condition (46) guarantees the fulfillment of the quantum regression hypothesis. This constraint is satisfied, for example, by a two-level system with Liouvillian $\mathcal{L}_{S}[\bullet]=-i \omega_{A}\left[\sigma_{z}, \bullet\right] / 2$, where $\sigma_{z}$ is the $z$-Pauli matrix, $\omega_{A}$ its transition frequency, and $\mathcal{E}$ (or equivalently $\mathcal{L}[\bullet]$ ) defines a dispersive or a thermal reservoir (see, respectively, Eqs. (71) and (74) in Ref. [7]). When the two-level system is subjected to an external field, the regression hypothesis may be broken. In fact, when condition (46) is not fulfilled, the regression hypothesis does not hold true in any case. Nevertheless, by writing

$$
\mathcal{L}_{S}=\mathcal{L}_{0}+\epsilon \mathcal{L}_{1}
$$

where $\left[\mathcal{L}_{0}, \mathcal{E}\right]=0$ and $\left[\mathcal{L}_{1}, \mathcal{E}\right] \neq 0$, it is possible to prove that-to first order in the parameter $\epsilon$ - the regression hy- 
pothesis, independent of the specific structure of $\mathcal{L}_{1}$, is still valid. In the Schrödinger representation, the operator expectation values $[\mathrm{Eq} .(55)]$ and the operator correlations $[\mathrm{Eq}$. (56)], to first order in $\epsilon$, read

$$
\begin{aligned}
& \overline{\mathbf{A}(\tau)} \approx \operatorname{Tr}_{S}\left\{\mathbf{A} \mathcal{G}(u)\left[\rho_{S}(z)+\mathrm{I}_{\rho}(u, z)\right]\right\}, \\
& \overline{O(t) \mathbf{A}(t+\tau)} \approx \operatorname{Tr}_{S}\left\{\mathbf{A} \mathcal{G}(u)\left[\rho_{S}(z)+\mathrm{I}_{\rho}(u, z)\right] O\right\} .
\end{aligned}
$$

Note that $\mathcal{G}(u)$ and $\mathrm{I}_{\rho}(u, z)$ are defined by Eqs. (16) and (17), respectively. After performing the preparation at time $t$, the inhomogeneous term follows from Eq. (30).

The work of Ref. [16] discussed the validity of the regression hypothesis in the context of non-Markovian dynamics based on the Lindblad rate equations [15]. It was also found (see Sec. 6) that an external (noncommuting) field breaks its applicability. Nevertheless, in contrast with the present formalism, the non-Markovian effects admit an underlying Markovian description. Furthermore, the regression theorem was studied by analyzing the dynamics at the initial and at the asymptotic times. In that case, the vanishing of the inhomogeneous term is a necessary condition for the validity of the regression hypothesis. In spite these differences, both formalisms lead to consistent and noncontradictory results.

We remark that similar conditions but not equivalent to Eq. (46) were found in different contexts. In Refs. [46-48] the validity of the quantum regression hypothesis beyond a weak-coupling regime was discussed [16]. In Ref. [49], a commutation property between the system-bath interaction and the system operators was derived by using a stochastic wave-vector formalism and taking into account a bosonic bath described in a rotating wave approximation. All these results suggest that, beyond a Markovian regime, the validity of the quantum regression hypothesis strongly may depend on the underlying microscopic dynamics. Nevertheless, the search for general applicable criteria should not be discarded [16].

\section{LINEAR-RESPONSE THEORY}

Here we analyze the response of the system, whose density-matrix evolution is given by Eq. (18), to an external time-dependent perturbation. In the stationary case [30,31], the system response to weak external perturbations is expressed in terms of response functions that are proportional to the cross correlation function between the variable of interest and a system variable coupled to the external field. Here, we show that a similar result can be established but that, nevertheless, strong departures from the predictions of the stationary theory may arise.

To simplify the analysis, in the following calculations we assume that $t=0$, i.e., that the system-environment coupling (initialization of the renewal dynamics) coincides with the preparation time and that the coupling with the external field is switched on at the same time. In this case the absolute time coincides with the distance $\tau$ from the system-environment coupling. The average system state $\rho_{S}(\tau)$ is written as a series in the external perturbation

$$
\rho_{S}(\tau) \simeq \rho_{S}^{(0)}(\tau)+\lambda \rho_{S}^{(1)}(\tau)+\cdots .
$$

The parameter $\lambda$ measures the strength of the external perturbation. $\rho_{S}^{(0)}(\tau)$ corresponds to the dynamics in the absence of the perturbation. The contribution $\rho_{S}^{(1)}(\tau)$ can be obtained from an average of the perturbed realizations or from the (perturbed) master equation defining the density-matrix evolution [Eq. (18)]. In general, one may assume that the external perturbation affects either the unitary or the dissipative dynamics. Hereby, we analyze both cases.

\section{A. Perturbing the dissipative dynamics}

Here, we consider the case when the external perturbation affects (or is coupled to) the dissipative dynamics. The two contributions in Eq. (65) are evaluated by averaging the perturbed stochastic realizations. The zeroth-order contribution reads

$$
\rho_{S}^{(0)}(\tau)=\sum_{n=0}^{\infty} \int_{0}^{\tau} d \tau^{\prime} \mathcal{P}_{0}\left(\tau-\tau^{\prime}\right) \mathcal{W}^{(n)}\left(\tau^{\prime}\right) \rho_{S}(0)
$$

Notice that this expression follows straightforwardly from Eq. (2). The first-order contribution is determined by an average over all possible trajectories, in each of which the external perturbation acts only once to fit the request of a linear response. Then, $\rho_{S}^{(1)}(\tau)$ becomes the double sum

$$
\begin{aligned}
\rho_{S}^{(1)}(\tau)= & \sum_{n=0}^{\infty} \sum_{m=0}^{\infty} \int_{0}^{\tau} d \tau_{1} \int_{0}^{\tau_{1}} d \tau_{2} \int_{0}^{\tau_{2}} d \tau_{3} \mathcal{P}_{0}\left(\tau-\tau_{1}\right) \\
& \times \mathcal{W}^{(n)}\left(\tau_{1}-\tau_{2}\right) \mathcal{O}\left(\tau_{2}, \tau_{3}\right) \mathcal{W}^{(m)}\left(\tau_{3}\right) \rho_{S}(0) .
\end{aligned}
$$

Each sum takes into account all possible events, preceding and ensuing the action of the external perturbation. In this expression, $\mathcal{P}_{0}(\tau)$ and $\mathcal{W}^{(n)}(\tau)$ are defined by Eqs. (3) and (4), respectively. The influence of the external perturbation is described by the superoperator $\mathcal{O}\left(\tau_{2}, \tau_{3}\right)$. This is done either by making the superoperator $\mathcal{E}$ change with time, without affecting the times of event occurrence, or by allowing the external stimulus to slightly change the times of event occurrence, namely, a little bit earlier or later, according to the system's state.

\section{Perturbing the event superoperator}

In Ref. [36] the linear-response theory was analyzed on the basis of a classical two-level system where the perturbation does not affect the time of occurrence of an event, but that the coin tossing selecting the fluctuations sign has a time-dependent bias. In Sec. II we have seen that the Krauss operators $\mathcal{E}[\rho]$ signal the occurrence of renewal events. To realize a perturbation on the system of the same nature, we have to assume that the time of occurrence of collisional events is not affected by the external perturbation, but that the specific form of $\mathcal{E}[\rho]$ is. Then, the Krauss operator is written as

$$
\mathcal{E}(\tau)[\rho]=\mathcal{E}[\rho]+\lambda \mathcal{O}(\tau)[\rho],
$$

where the superoperator $\mathcal{O}(\tau)$ satisfies $\operatorname{Tr}_{S}\{\mathcal{O}(t)[\rho]\}=0$. For simplicity, we assume 


$$
\mathcal{O}(\tau)=\xi(\tau) \mathcal{O}
$$

$\xi(\tau)$ is a scalar function that defines the temporal dependence of the external perturbation. The superoperator $\mathcal{O}\left(\tau_{2}, \tau_{3}\right)$ appearing in Eq. (67) can then be written as

$$
\mathcal{O}\left(\tau_{2}, \tau_{3}\right)=\mathcal{O}\left(\tau_{2}\right) \exp \left[\left(\tau_{2}-\tau_{3}\right) \mathcal{L}_{S}\right] w\left(\tau_{2}-\tau_{3}\right) .
$$

By working in the Laplace domain on the contributions to each sum of Eq. (67), after some algebra, we get

$$
\begin{aligned}
\rho_{S}(\tau) \simeq & \mathcal{G}(\tau) \rho_{S}(0)+\lambda \int_{0}^{\tau} d \tau^{\prime} \mathcal{G}\left(\tau-\tau^{\prime}\right) \mathcal{O}\left(\tau^{\prime}\right) \\
& \times \int_{0}^{\tau^{\prime}} d \tau^{\prime \prime} K\left(\tau^{\prime}-\tau^{\prime \prime}\right) e^{\left(\tau^{\prime}-\tau^{\prime \prime}\right) \mathcal{L}_{S}} \mathcal{G}\left(\tau^{\prime \prime}\right) \rho_{S}(0)
\end{aligned}
$$

The propagator $\mathcal{G}(\tau)$ is defined in the Laplace domain by Eq. (16), while the kernel $K(\tau)$ is defined by Eq. (21).

Equation (71) generates the system's response to first order in the perturbation strength $\lambda$ and consequently the system's linear response. For the operator expectation values, after introducing assumption (69), we get

$$
\overline{\mathbf{A}(\tau)}=\overline{\mathbf{A}_{0}(\tau)}+\lambda \int_{0}^{\tau} d \tau^{\prime} \chi_{\mathbf{A} \mathcal{O}}\left(\tau, \tau^{\prime}\right) \xi\left(\tau^{\prime}\right),
$$

where the zeroth-order contribution reads $\overline{\mathbf{A}_{0}(\tau)}$ $=\operatorname{Tr}_{S}\left[\mathbf{A} \mathcal{G}(\tau) \rho_{S}(0)\right]$, and the response function is given by

$$
\chi_{\mathbf{A} \mathcal{O}}\left(\tau, \tau^{\prime}\right)=\operatorname{Tr}_{S}\left[\mathbf{A} \mathcal{G}\left(\tau-\tau^{\prime}\right) \mathcal{O} \rho_{f}\left(\tau^{\prime}\right)\right] .
$$

With $\rho_{f}\left(\tau^{\prime}\right)$, we denote

$$
\rho_{f}\left(\tau^{\prime}\right)=\int_{0}^{\tau^{\prime}} d \tau^{\prime \prime} K\left(\tau^{\prime}-\tau^{\prime \prime}\right) e^{\left(\tau^{\prime}-\tau^{\prime \prime}\right) \mathcal{L}_{S}} \mathcal{G}\left(\tau^{\prime \prime}\right) \rho_{S}(0) .
$$

Evidently, the response function $\chi_{\mathbf{A O}}\left(\tau, \tau^{\prime}\right)$ has the structure of an operator correlation [see Eq. (57)]. This fact becomes more evident when the initial density matrix corresponds to the stationary state $\rho_{S}^{\infty}$ of the unperturbed evolution, i.e.,

$$
\rho_{S}^{\infty} \equiv \lim _{\tau \rightarrow \infty} \mathcal{G}(\tau) \rho_{S}(0),
$$

and the condition

$$
\mathcal{L}_{S}\left[\rho_{S}^{\infty}\right]=\rho_{S}^{\infty}
$$

is satisfied. Then, the expectation values are written as

$$
\overline{\mathbf{A}(\tau)}=\overline{\mathbf{A}_{\infty}}+\lambda \int_{0}^{\tau} d \tau^{\prime} \chi_{\mathbf{A} \mathcal{O}}^{\infty}\left(\tau, \tau^{\prime}\right) \xi\left(\tau^{\prime}\right),
$$

where $\overline{\mathbf{A}_{\infty}}=\operatorname{Tr}_{S}\left[\mathbf{A} \rho_{S}^{\infty}\right]$. The response function becomes

$$
\chi_{\mathbf{A} \mathcal{O}}^{\infty}\left(\tau, \tau^{\prime}\right)=\operatorname{Tr}_{S}\left[\mathbf{A} \mathcal{G}\left(\tau-\tau^{\prime}\right) \mathcal{O} \rho_{S}^{\infty}\right] f\left(\tau^{\prime}, 0\right) .
$$

Here, the function $f(\tau, 0)$ is defined by Eq. (27). The quantum-statistical average over $\rho_{S}^{\infty}$ can be read as a correlation between the operator $\mathbf{A}$ and the superoperator $\mathcal{O}$ [see Eq. (57) with $z \rho_{S}(z) \rightarrow \rho_{S}^{\infty}$ and $\left.\Delta(u, z) \rightarrow 0\right]$.

For the Markovian dynamics [Eq. (28)], where $f\left(\tau^{\prime}, 0\right)$ $\rightarrow \gamma$, the response $\chi_{\mathbf{A} \mathcal{O}}^{\infty}\left(\tau, \tau^{\prime}\right)$ depends only on $\left(\tau-\tau^{\prime}\right)$. This stationary condition $[30,31]$ is broken in the non-Markovian case. In fact, the presence of the factor $f\left(\tau^{\prime}, 0\right)$ implies that $\chi_{\mathbf{A} \mathcal{O}}^{\infty}\left(\tau, \tau^{\prime}\right)$ depends separately on both $\tau$ and $\tau^{\prime}$. Depending on the behavior of $f\left(\tau^{\prime}, 0\right)$ [see Eqs. (37) and (41)] in the long-time regime, the system may become insensitive to the external perturbation. This effect, which is sometimes called death of linear response [38], was found in classical systems in Refs. [32,37]. The present analysis leads us to conclude that the same amazing phenomenon may be observed in quantum systems.

The previous results also follows from calculations based on the density-matrix evolution [Eq. (18)]. On the other hand, we assumed that the external perturbation is switched on at the initial time. The general case, i.e., when the renewal dynamics start at time $t=0$ and the external perturbation is switched on at time $t>0$, can be discussed following the same calculations steps. The final expressions involve some extra contributions. Nevertheless, under assumption (62), to first order in $\epsilon$, Eq. (71) remains valid under the replacements $\rho_{S}(0) \rightarrow \rho_{S}(t) \quad$ and $\quad K(\tau) \rightarrow K_{t}(\tau)$, where $K_{t}(u)$ $=u \tilde{w}(u, t) /[1-w(u)]$. Similarly, Eq. (78) remains valid under the replacement $f\left(\tau^{\prime}, 0\right) \rightarrow f\left(\tau^{\prime}, t\right)$.

As an example we consider a two-level system, with states $\{| \pm\rangle\}$, whose unitary evolution is defined by $\mathcal{L}_{S}[\rho]=$ $-i \Omega\left[\sigma_{x}, \rho\right] / 2$, where $\sigma_{x}$ is the $x$-Pauli matrix in the basis $\{| \pm\rangle\}$. The perturbed superoperator reads

$$
\mathcal{E}(\tau)[\rho]=\frac{1}{2} \sum_{a, b} \sigma_{a b} \rho \sigma_{a b}^{\dagger}[1-a \lambda \xi(\tau)],
$$

where $\sigma_{a b} \equiv|a\rangle\langle b|,(a, b)= \pm$, and $\lambda|\xi(\tau)| \leq 1$. It can be rewritten as $\mathcal{E}(\tau)[\rho]=\left[\mathrm{I}+\lambda \xi(\tau) \sigma_{z}\right] / 2$. Then, both the unperturbed and the perturbed terms turn out to be independent of the initial state $\rho$. The unperturbed dynamics corresponds to a depolarizing channel [3], with stationary state $\rho_{S}^{\infty}=\mathrm{I} / 2$. The perturbed dynamics modulates the probability of transitions between the two states [36].

From Eq. (77), the difference between the upper and the lower populations, i.e., the mean value of the $z$-Pauli matrix $\sigma_{z}, S_{Z}(\tau)=\operatorname{Tr}_{S}\left[\rho_{S}(\tau) \sigma_{z}\right]$, reads

$$
S_{Z}(\tau)=\lambda \int_{0}^{\tau} d \tau^{\prime} P_{0}\left(\tau-\tau^{\prime}\right) \cos \left[\Omega\left(\tau-\tau^{\prime}\right)\right] f\left(\tau^{\prime}, 0\right) \xi\left(\tau^{\prime}\right)
$$

As the transformation $\mathcal{E}(\tau)[\rho]$ does not depend on $\rho$, it is simple to prove that Eq. (80) also corresponds to the exact solution to all orders in $\lambda$. On the other hand, notice that the results obtained in Ref. [36] are recovered in the limit $\Omega$ $\rightarrow 0$. Using Eq. (79), it is easy to establish the nature of the stochastic dynamics associated with Eq. (80), i.e., $S_{Z}(\tau)$ $=\left\langle S_{s t}(\tau)\right\rangle$, where $\langle\cdots\rangle$ denotes the average over the single realizations. Between two consecutive events [action of $\mathcal{E}(\tau)]$, occurring at times $\tau_{i-1}$ and $\tau_{i}$, the stochastic evolution is given by $S_{s t}(\tau)=\cos \left[\Omega\left(\tau-\tau_{i-1}\right)\right] S_{s t}\left(\tau_{i-1}\right)$, where $\tau$ $\in\left(\tau_{i}, \tau_{i-1}\right)$, while at $\tau=\tau_{i}$, we apply the disruptive transformation $S_{s t}(\tau) \rightarrow \lambda \xi\left(\tau_{i}\right)$. The statistics of the time intervals $\left(\tau_{i}-\tau_{i-1}\right)$ is given by the distribution $w(\tau)$. In the result illustrated by the following figures, we use Eq. (40). 

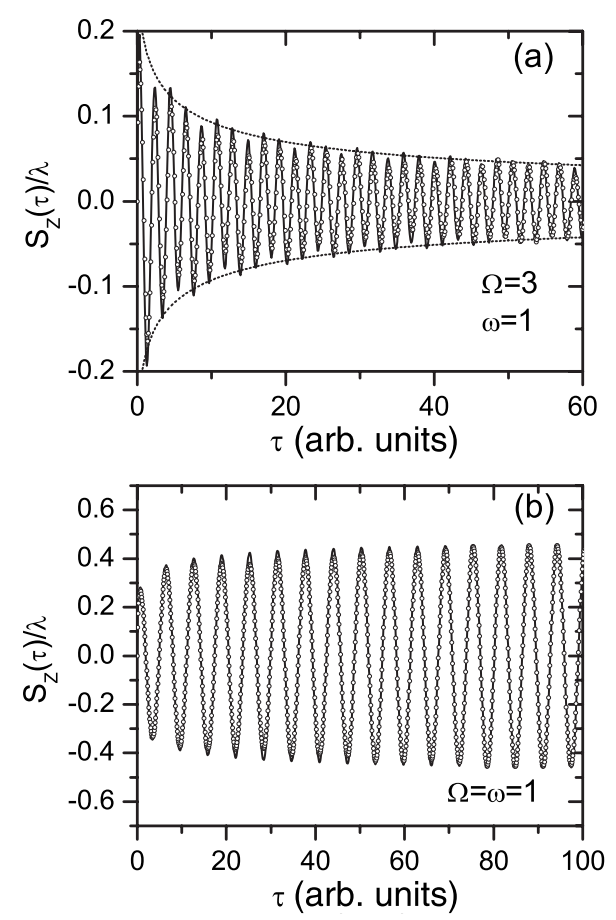

FIG. 3. Mean value of the $z$-Pauli matrix (full line), Eq. (80), driven by a periodic external perturbation, Eq. (79), with $\xi(\tau)$ $=\cos [\omega \tau]$. The parameters of the waiting-time distribution, Eq. (40), are $\alpha=1 / 2$ and $A_{\alpha}=1 / 2$. The strength of the perturbation is $\lambda$ $=0.1$. The circles correspond to an average over $10^{3}$ realizations (see text). In (a) the parameters are $\Omega=3$ and $\omega=1$. The dotted lines are proportional to $\pm P_{0}(\tau)$. In (b), $\Omega=\omega=1$. The time $\tau$ and the parameters are measured in arbitrary units.

In Fig. 3 we show both the solution of Eq. (80) and the average on the realizations of the stochastic simulation. The function $f(\tau, 0)$ is given by Eq. (41). The external perturbation is $\xi(\tau)=\cos [\omega \tau]$. We note that, in general, in the longtime regime the system becomes insensitive to the external perturbation [Fig. 3(a)]. In fact, in the Markovian case, or when $\lim _{\tau \rightarrow \infty} f(\tau, 0)>0$, the asymptotic behavior of the mean value $\lim _{\tau \rightarrow \infty} S_{Z}(\tau)$ is given by an oscillatory function. In contrast, here, $\lim _{\tau \rightarrow \infty} S_{Z}(\tau)=0$. The decay to this asymptotic value is given by a power-law function. The oscillation amplitude is proportional to the survival probability [Eq. (5)], which can be written here as $P_{0}(\tau)$ $=\exp \left[A_{1 / 2}^{2} t\right] \operatorname{erfc}\left[A_{1 / 2} t^{1 / 2}\right]$. In the time asymptotic regime, it behaves as $P_{0}(\tau) \approx 1 /\left(A_{1 / 2} \sqrt{t}\right)$ [44]. Only when $\omega=\Omega$ the asymptotic behavior is given by an undamped oscillatory function [Fig. 3(b)]. The presence of an undamped asymptotic contribution follows straightforwardly from Eq. (80) after expanding the involved trigonometric functions and using $P_{0}(u) f(u, 0)=1 / u-P_{0}(u)$. The nonvanishing contribution is $\left[1-P_{0}(\tau)\right] \cos [\omega \tau] / 2$. Thus, the convergence to the maximal amplitude oscillation (one half) also follows a power-law behavior. This effect is seen in Fig. 3(b).

\section{Perturbing the times of event occurrences}

In Refs. [33,32], the response of a classical two-level system was analyzed by assuming that the external perturbation affects the times of event occurrence. The prediction gener- ated by this assumption has been recently confirmed by experimental results on liquid crystals [41]. Here, that assumption corresponds to assuming that the times of the superoperator $\mathcal{E}$ 's action are slightly changed by the external perturbation. Below, we discuss a system-bath modeling where this condition applies.

We consider a system, which may also have its own (Markovian) dissipative dynamics, and whose interaction with a complex bath only occurs when the environment undergoes a structural change, implying the application of $\mathcal{E}$ over the system density matrix. The changes between the different structures of the bath are described by a complex landscape. The escape over a single well is described by the standard Kramers theory. By turning on an external perturbation, the height $V$ of a given well is written as $V(t)=V_{0}+\lambda_{0} \xi(t)$. By assuming an adiabatic regime, the survival probability associated with each well evolves as

$$
\frac{d}{d \tau} P_{0}(\tau \mid t) \simeq-\gamma_{0}(\tau+t) P_{0}(\tau \mid t) .
$$

Here, $P_{0}(\tau \mid t)$ defines the conditional probability that no event (structural change) occurs in the time interval $(t, t+\tau)$ given that the last event occurred at time $t$. The timedependent rate is written as $\gamma_{0}(t)=\gamma_{0} \exp [-V(t) / D]$. The coefficient $D$ involves the temperature and extra parameters describing the well shape. The solution of Eq. (81) reads $P_{0}(\tau \mid t) \simeq \exp \left[-\int_{0}^{\tau} \gamma_{0}\left(\tau^{\prime}+t\right) d \tau^{\prime}\right]$. To first order in $\lambda_{0}$, it follows that

$$
P_{0}(\tau \mid t) \simeq \exp \left\{-\gamma\left[\tau+\lambda \int_{t}^{t+\tau} \xi\left(t^{\prime}\right) d t^{\prime}\right]\right\},
$$

where $\gamma=\gamma_{0} \exp \left[-V_{0} / D\right]$ is the Kramers rate and the dimensionless strength parameter reads $\lambda=-\lambda_{0} / D$. If one assumes a statistical distribution of rate $\gamma$ (due to a random $\gamma_{0}$ or $V_{0}$ ), the survival probability $P_{0}(\tau \mid t)$ must be written as a statistical superposition of exponential functions, from which arbitrary decay behaviors can be recovered. Therefore, Eq. (82) can be extended to nonexponential survival probabilities.

In conclusion, the external perturbation shifts the time of event occurrence, and this property, on the basis of the earlier arguments corresponds to the assumption

$$
P_{0}(\tau \mid t)=P_{0}\left[\tau+\lambda \mathcal{O} \int_{t}^{t+\tau} d t^{\prime} \xi\left(t^{\prime}\right)\right] .
$$

The function $\xi\left(t^{\prime}\right)$ defines the time dependence of the perturbation. The superoperator $\mathcal{O}$ takes into account a dependence of the time shift on the system state.

To first order in $\lambda$, the survival probability reads

$$
P_{0}(\tau \mid t) \approx P_{0}(\tau)-\lambda \mathcal{O} w(\tau) \int_{t}^{t+\tau} d t^{\prime} \xi\left(t^{\prime}\right)
$$

Although, in principle, the external perturbation breaks the renewal character of the process, under the assumption of weak perturbation (small $\lambda$ ), it is legitimate to define the conditional waiting-time distribution density $w(\tau \mid t)$ $=-(\partial / \partial \tau) P_{0}(\tau \mid t)$, thereby getting 


$$
w(\tau \mid t) \approx w(\tau)+\lambda \mathcal{O} \frac{\partial}{\partial \tau}\left[w(\tau) \int_{t}^{t+\tau} d t^{\prime} \xi\left(t^{\prime}\right)\right] .
$$

Notice that under this approximation, independent of the $\mathcal{O}$ structure, the normalization condition $\int_{0}^{\infty} d \tau w(\tau \mid t)=1$ is preserved.

The earlier assumptions do not affect Eq. (67), which is made to remain valid by defining $\mathcal{O}\left(\tau_{2}, \tau_{3}\right)$ as

$$
\mathcal{O}\left(\tau_{2}, \tau_{3}\right)=\mathcal{O}^{\prime} \exp \left[\left(\tau_{2}-\tau_{3}\right) \mathcal{L}_{S}\right] \delta w\left(\tau_{2} \mid \tau_{3}\right),
$$

where $\mathcal{O}^{\prime}=\mathcal{E} \mathcal{O}$ and

$$
\delta w\left(\tau_{2} \mid \tau_{3}\right)=\frac{\partial}{\partial \tau_{2}}\left[w\left(\tau_{2}-\tau_{3}\right) \int_{\tau_{3}}^{\tau_{2}} d t^{\prime} \xi\left(t^{\prime}\right)\right] .
$$

Nevertheless, in this case, the operator $\mathcal{P}_{0}\left(\tau-\tau_{1}\right)$ appearing in Eq. (67) also gives a contribution to first order in $\lambda$, as clearly shown by Eq. (84).

By assuming the initial condition (75), the condition of Eq. (76), doing the same calculus for the derivation of Eq. (71), and after rearranging the time integrals and some algebra, the operator expectation values read

$$
\overline{\mathbf{A}(\tau)}=\overline{\mathbf{A}_{\infty}}+\lambda \int_{0}^{\tau} d \tau^{\prime} \chi_{\mathbf{A} \mathcal{O}}^{\infty}\left(\tau, \tau^{\prime}\right) \xi\left(\tau^{\prime}\right),
$$

with $\overline{\mathbf{A}_{\infty}}=\operatorname{Tr}_{S}\left[\mathbf{A} \rho_{S}^{\infty}\right]$ and the response function being

$$
\begin{aligned}
\chi_{\mathbf{A} \mathcal{O}}^{\infty}\left(\tau, \tau^{\prime}\right)= & \widetilde{w}\left(\tau-\tau^{\prime}, \tau^{\prime}\right) \operatorname{Tr}_{S}\left\{\mathbf{A}[\mathcal{E}, \mathcal{O}] \rho_{S}^{\infty}\right\} \\
& -\int_{\tau^{\prime}}^{\tau} d \tau^{\prime \prime} \widetilde{w}\left(\tau^{\prime \prime}-\tau^{\prime}, \tau^{\prime}\right) \frac{\partial}{\partial \tau^{\prime \prime}} \\
& \times\left\{\operatorname{Tr}_{S}\left[\mathbf{A} \mathcal{G}\left(\tau-\tau^{\prime \prime}\right) \mathcal{O}^{\prime} \rho_{S}^{\infty}\right]\right\},
\end{aligned}
$$

where $\widetilde{w}\left(\tau-\tau^{\prime}, \tau^{\prime}\right)$ is defined by Eq. (24). Thus, in this case the response function is also proportional to the correlation between the operator $\mathbf{A}$ and the external perturbation $\mathcal{O}$.

When $\mathcal{O}^{\prime} \rho_{S}^{\infty}=\rho_{S}^{\infty}$, or $\mathcal{O}^{\prime} \rho_{S}^{\infty}=0$, the integral contribution to Eq. (89) vanishes. Then Eq. (88) yields

$$
\overline{\mathbf{A}(\tau)}=\overline{\mathbf{A}_{\infty}}+\lambda \overline{\mathbf{A}_{\infty}^{\prime}} \int_{0}^{\tau} d \tau^{\prime} \widetilde{w}\left(\tau-\tau^{\prime}, \tau^{\prime}\right) \xi\left(\tau^{\prime}\right),
$$

where $\overline{\mathbf{A}_{\infty}^{\prime}},=\operatorname{Tr}_{S}\left\{\mathbf{A}[\mathcal{E}, \mathcal{O}] \rho_{S}^{\infty}\right\}$. With the earlier arguments in mind, we state that this kind of response function is generated whenever the underlying dynamics can be modeled as an escape process from a well through time-dependent barrier, or in general when prescription (83) applies. This makes it possible to use for $w(\tau)$ any form and, not necessarily, the inverse power-law form of Refs. [32,33]. The example discussed in these papers is a symmetrical two-level system, with $\mathcal{E}[\rho]=\Sigma_{a, b= \pm} \sigma_{a b} \rho \sigma_{a b}^{\dagger} / 2=\mathrm{I} / 2, \quad \rho_{S}^{\infty}=\mathrm{I} / 2, \mathcal{O}[\rho]$ $=-\Sigma_{a= \pm} a \sigma_{a a} \rho \sigma_{a a}^{\dagger}, \mathcal{L}_{S}=0$, and $\mathbf{A} \rightarrow \sigma_{z}$. In this case, $\chi_{\mathbf{A} \mathcal{O}}^{\infty}\left(\tau, \tau^{\prime}\right)$ follows straightforwardly from the first-order contribution associated with Eq. (84). On the other hand, while Eqs. (78) and (89) define the system response in terms of operator correlations, they do not involve in general the derivative of an operator correlation [34].

\section{B. Perturbing the unitary dynamics}

Now we consider the case where the external perturbation affects the unitary dynamics acting in the time interval between the occurrences of two consecutive events. Then, we write

$$
\mathcal{L}_{S}(\tau)=\mathcal{L}_{S}+\lambda \mathcal{L}_{\text {ext }}(\tau)
$$

In contrast to the previous case, here, we show that when the dynamics strongly departs from the Markovian case, it is not possible to generate a linear-response theory.

The perturbed dynamics [Eq. (65)] are derived from the master equation defining the density-matrix evolution [Eq. (18)] with $t=0$. To first order in $\lambda$, we get

$$
\frac{d \rho_{S}(\tau)}{d \tau} \simeq \mathcal{L}_{S}(\tau) \rho_{S}(\tau)+\int_{0}^{\tau} d \tau^{\prime} K\left(\tau-\tau^{\prime}\right) \mathcal{L U}\left(\tau, \tau^{\prime}\right) \rho_{S}\left(\tau^{\prime}\right)
$$

Here, $\mathcal{U}\left(\tau^{\prime}, \tau^{\prime \prime}\right)$ is a propagator associated with the timedependent Liouvillian superoperator (91), i.e., $\mathcal{U}\left(\tau^{\prime}, \tau^{\prime \prime}\right)$ $=\exp \left[\mathcal{L}_{S}\left(\tau^{\prime}-\tau^{\prime \prime}\right)\right]+\lambda \mathcal{U}^{(1)}\left(\tau^{\prime}, \tau^{\prime \prime}\right)+\cdots$, where $\mathcal{U}^{(1)}\left(\tau^{\prime}, \tau^{\prime \prime}\right)$ is the first-order contribution.

The first-order contribution in Eq. (65), by assuming the stationary initial condition (75), reads

$$
\begin{aligned}
\rho_{S}^{(1)}(\tau)= & \int_{0}^{\tau} d \tau^{\prime} \mathcal{G}\left(\tau-\tau^{\prime}\right)\left\{\mathcal{L}_{\mathrm{ext}}\left(\tau^{\prime}\right) \rho_{S}^{\infty}\right. \\
& \left.+\int_{0}^{\tau^{\prime}} d \tau^{\prime \prime} K\left(\tau^{\prime}-\tau^{\prime \prime}\right) \mathcal{L} \mathcal{U}^{(1)}\left(\tau^{\prime}, \tau^{\prime \prime}\right) \rho_{S}^{\infty}\right\} .
\end{aligned}
$$

When calculating the operator expectation values, the first line recovers the standard Kubo response theory [31]. On the other hand, the second line shows that it is impossible to generate a first-order perturbation. In fact, the validity of this contribution relies on approximating the difference between the perturbed and the unperturbed propagators, $\mathcal{U}\left(\tau^{\prime}, \tau^{\prime \prime}\right)$ $-\exp \left[\mathcal{L}_{S}\left(\tau^{\prime}-\tau^{\prime \prime}\right)\right]$, by the first-order contribution $\lambda \mathcal{U}^{(1)}\left(\tau^{\prime}, \tau^{\prime \prime}\right)$. Nevertheless, if the kernel $K\left(\tau^{\prime}-\tau^{\prime \prime}\right)$ correlates distant times $\left(\tau^{\prime}\right.$ and $\left.\tau^{\prime \prime}\right)$, evidently $\mathcal{U}\left(\tau^{\prime}, \tau^{\prime \prime}\right)$ cannot be approximated to first order in $\lambda$. Only when the non-Markovian dynamics slightly depart from the Markovian condition $\left[K\left(\tau^{\prime}-\tau^{\prime \prime}\right)=\gamma \delta\left(\tau^{\prime}-\tau^{\prime \prime}\right)\right]$ the perturbed dynamics can be approximated to first order in the perturbation. The same conclusion follows by analyzing the perturbed stochastic trajectories.

\section{SUMMARY AND CONCLUSIONS}

In this paper we have shown that nonstandard nonstationary statistical effects can arise in the context of CP openquantum-system dynamics. The results rely on modeling the system dynamics through a renewal approach, where the density matrix follows after averaging a set of realizations that mimic the interaction with a non-Markovian environment. The realizations are characterized by disruptive abrupt events, producing changes described by the application of a $\mathrm{CP}$ superoperator. The time distance between the occurrences of two consecutive collisional events is drawn from a nonPoissonian waiting-time distribution density $w(\tau)$. In the 
time intervals between two consecutive collisional events, the system's time evolution is described by a unitary prescription. Both the $\mathrm{CP}$ superoperator and the waiting-time distribution density take into account the interaction of the system with the environment.

As a significant advancement compared to the earlier work, here, we analyzed the non-Markovian system dynamics by introducing a system preparation at an arbitrary time and studied the ensuing evolution. The preparation erases the dependence of the evolution on the previous history of the system. Nevertheless, it does not erase the memory of the universe, i.e., the system-environment arrangement. In fact, the master evolution after preparation depends explicitly on the time preparation [Eqs. (18) and (30) or Eq. (31)]. When the preparation time is done at arbitrary long times, the ensuing density-matrix evolution may or may not converge to an asymptotic structure, with the last situation defining the nonstationary case. It arises when the average time between events is divergent. When there exists an asymptotic stationary evolution, we showed that it may significantly depart from the evolution ensuing preparation at the initial time, i.e., in general the stationary evolution may develop stronger or weaker non-Markovian effects than the evolution ensuing the preparation at the initial time.

The possibility of extending the regression hypothesis to the evolutions arising from the renewal approach was also explored. We showed that, when the unitary dynamics commutes with the event superoperator, the non-Markovian evolutions of the expectation and the correlation operators are exactly the same [Eqs. (55) and (56)]. This result is valid even in the presence of nonstationary effects. When the commutation condition is not satisfied, the regression hypothesis remains valid [Eqs. (63) and (64)] up to first order in the perturbation [Eq. (62)].

The nonstationary character of the evolution was also analyzed through the response of the system to an external weak perturbation. When the external field modifies the dissipative dynamics, the response function associated with the mean value of a given operator can be written as a function of the correlation between the operator and the external perturba- tion. Different response functions [Eqs. (78) and (89)] are generated depending on whether we make the perturbation modifies the superoperator structure [Eq. (68)] without affecting the occurrence time of the collisional events or we make the perturbation affects the time occurrence of the disruptive events [Eq. (83)]. As in the classical counterparts, we have shown that, in the presence of nonstationary dynamics, the response of the system may die out in the time asymptotic regime. We also concluded that when the external perturbation modifies the unitary dynamics between events, the linear-response theory is incompatible with the presence of strong memory effects.

The equations that express the previous results are also valid for classical systems. In fact, all quantum properties disappear if one disregards the unitary contributions, considers diagonal density matrices, and takes superoperators that do not break that condition. With respect to previous analyses $[28,29,32-35]$, the present results do not rely on a specific form of the waiting-time distribution (such as the inverse power-law forms) neither rely on a classical two-level system modeling. Thus, the formalism applies even when the events are defined by differential (Fokker-Planck) operators.

The results found in this paper may have direct experimental implications. In fact, the renewal dynamics arises trivially in the context of (non-Markovian) quantum kicked systems [50]. The main conclusions arrived at with our analysis may also apply to quantum systems coupled to complex reservoirs generating decay behaviors without a characteristic time scale $[15,21]$. While a full quantum microscopic derivation of the present results is an open problem, our analysis demonstrates that a rich kind of behaviors may arise when dealing with non-Markovian nonstationary quantum evolutions. Our contribution is a consistent attempt to model those issues in the context of CP open-quantum-system dynamics.

\section{ACKNOWLEDGMENTS}

The authors thank Welch Foundation for financial support of this work through Grant No. B-1577. A.A.B. also thanks support from CONICET, Argentina.
[1] H. P. Breuer and F. Petruccione, The Theory of Open Quantum Systems (Oxford University Press, Oxford, 2002).

[2] H. J. Carmichael, An Open Systems Approach to Quantum Optics, Lecture Notes in Physics Vol. M18 (Springer, Berlin, 1993).

[3] M. A. Nielsen and I. L. Chuang, Quantum Computation and Quantum Information (Cambridge University Press, Cambridge, England, 2000).

[4] U. Weiss, Quantum Dissipative Systems (World Scientific, Singapore, 1999); F. Haake, Statistical Treatment of Open Systems by Generalized Master Equations (Springer, New York, 1973).

[5] S. M. Barnett and S. Stenholm, Phys. Rev. A 64, 033808 (2001).

[6] J. Wilkie, Phys. Rev. E 62, 8808 (2000); J. Chem. Phys. 114, 7736 (2001); 115, 10335 (2001); J. Wilkie and Y. M. Wong, J.
Phys. A 42, 015006 (2009).

[7] A. A. Budini, Phys. Rev. A 69, 042107 (2004).

[8] S. Daffer, K. Wodkiewicz, J. D. Cresser, and J. K. McIver, Phys. Rev. A 70, 010304(R) (2004); E. Andersson, J. D. Cresser, and M. J. V. Hall, J. Mod. Opt. 54, 1695 (2007).

[9] J. Salo, S. M. Barnett, and S. Stenholm, Opt. Commun. 259, 772 (2006).

[10] A. Shabani and D. A. Lidar, Phys. Rev. A 71, 020101(R) (2005).

[11] S. Maniscalco, Phys. Rev. A 75, 062103 (2007); 72, 024103 (2005); S. Maniscalco and F. Petruccione, ibid. 73, 012111 (2006).

[12] A. Kossakowski and R. Rebolledo, Open Syst. Inf. Dyn. 14, 265 (2007); 15, 135 (2008).

[13] H. P. Breuer and B. Vacchini, Phys. Rev. Lett. 101, 140402 
(2008).

[14] R. S. Whitney, J. Phys. A 41, 175304 (2008).

[15] A. A. Budini, Phys. Rev. A 74, 053815 (2006); Phys. Rev. E 72, 056106 (2005); A. A. Budini and H. Schomerus, J. Phys. A 38, 9251 (2005).

[16] A. A. Budini, J. Stat. Phys. 131, 51 (2008).

[17] H. P. Breuer, Phys. Rev. A 75, 022103 (2007); H. P. Breuer, J. Gemmer, and M. Michel, Phys. Rev. E 73, 016139 (2006).

[18] J. Fischer and H. P. Breuer, Phys. Rev. A 76, 052119 (2007).

[19] B. Vacchini, Phys. Rev. A 78, 022112 (2008).

[20] M. Esposito and P. Gaspard, Phys. Rev. E 76, 041134 (2007).

[21] A. A. Budini, Phys. Rev. A 73, 061802(R) (2006); 76, 023825 (2007); J. Chem. Phys. 126, 054101 (2007); J. Phys. B 40, 2671 (2007); Phys. Rev. A 79, 043804 (2009).

[22] E. W. Montroll and G. H. Weiss, J. Math. Phys. 6, 167 (1965); H. Scher and E. W. Montroll, Phys. Rev. B 12, 2455 (1975); V. M. Kenkre, E. W. Montroll, and M. F. Shlesinger, J. Stat. Phys. 9, 45 (1973).

[23] X. Brokmann, J. P. Hermier, G. Messin, P. Desbiolles, J. P. Bouchaud, and M. Dahan, Phys. Rev. Lett. 90, 120601 (2003).

[24] G. Aquino, L. Palatella, and P. Grigolini, Phys. Rev. Lett. 93, 050601 (2004); S. Bianco, P. Grigolini, and P. Paradisi, J. Chem. Phys. 123, 174704 (2005); P. Allegrini, F. Barbi, P. Grigolini, and P. Paradisi, Phys. Rev. E 73, 046136 (2006).

[25] R. Verberk, J. W. M. Chon, M. Gu, and M. Orrit, Physica E (Amsterdam) 26, 19 (2005); F. D. Stefani, X. Zhong, W. Knoll, M. Han, and M. Kreiter, New J. Phys. 7, 197 (2005).

[26] P. Frantsuzov, M. Kuno, B. Janko, and R. A. Marcus, Nat. Phys. 4, 519 (2008); F. D. Stefani, J. P. Hoogenboom, and E. Barkai, Phys. Today 62(2), 34 (2009).

[27] L. Onsager, Phys. Rev. 37, 405 (1931); 38, 2265 (1931).

[28] P. Allegrini, G. Aquino, P. Grigolini, L. Palatella, A. Rosa, and B. J. West, Phys. Rev. E 71, 066109 (2005).

[29] P. Allegrini, G. Aquino, P. Grigolini, L. Palatella, and A. Rosa, Phys. Rev. E 68, 056123 (2003); G. Aquino, M. Bologna, P. Grigolini, and B. J. West, ibid. 70, 036105 (2004).

[30] R. Kubo, Rep. Prog. Phys. 29, 255 (1966).

[31] R. Kubo, M. Toda, and N. Hashitsume, Statistical Physics II
(Springer-Verlag, Berlin, 1999).

[32] F. Barbi, M. Bologna, and P. Grigolini, Phys. Rev. Lett. 95 , 220601 (2005).

[33] P. Allegrini, M. Bologna, P. Grigolini, and B. J. West, Phys. Rev. Lett. 99, 010603 (2007).

[34] G. Aquino, P. Grigolini, and B. J. West, EPL 80, 10002 (2007).

[35] P. Grigolini, Int. J. Bifurcation Chaos 18, 2709 (2008).

[36] I. M. Sokolov, Phys. Rev. E 73, 067102 (2006).

[37] I. M. Sokolov, A. Blumen, and J. Klafter, Physica A 302, 268 (2001); I. M. Sokolov and J. Klafter, Phys. Rev. Lett. 97, 140602 (2006)

[38] I. M. Sokolov and J. Klafter, Chaos, Solitons Fractals 34, 81 (2007).

[39] M. Lukovic and P. Grigolini, J. Chem. Phys. 129, 184102 (2008).

[40] G. Margolin and E. Barkai, J. Stat. Phys. 122, 137 (2006); A. Rebenshtok and E. Barkai, ibid. 133, 565 (2008).

[41] L. Silvestri, L. Fronzoni, P. Grigolini, and P. Allegrini, Phys. Rev. Lett. 102, 014502 (2009).

[42] M. Lax, Phys. Rev. 129, 2342 (1963); 157, 213 (1967).

[43] In previous papers $[28,29]$ the preparation time $t$ was denoted by $t_{a}$ and called aging time.

[44] R. Metzler and J. Klafter, Phys. Rep. 339, 1 (2000).

[45] For distribution (40), function (35) reads $\widetilde{P}_{0}(\tau, t)$ $=\sum_{k=0}^{\infty} \frac{\left[-A_{\alpha}(\tau+t)^{\alpha}\right]^{k}}{\Gamma(\alpha k+1)}\left[1+B_{k}(\tau, t)\right]$, with the coefficients $B_{k}(\tau, t)$ $=\frac{A_{\alpha}(\tau+t)^{\alpha}}{\Gamma(\alpha)} \beta\left[\frac{t}{\tau+t} ; \alpha, 1+k \alpha\right] . \quad \beta[x ; a, b]$ is the incomplete beta function.

[46] G. W. Ford and R. F. O'Connell, Phys. Rev. Lett. 77, 798 (1996).

[47] G. W. Ford and R. F. O'Connell, Opt. Commun. 179, 451 (2000); 179, 477 (2000).

[48] M. Lax, Opt. Commun. 179, 463 (2000).

[49] D. Alonso and I. de Vega, Phys. Rev. Lett. 94, 200403 (2005); Phys. Rev. A 75, 052108 (2007).

[50] H. Schomerus and E. Lutz, Phys. Rev. Lett. 98, 260401 (2007); Phys. Rev. A 77, 062113 (2008). 